\title{
A first principles study of thiol-capped Au nanoparticles: Structural, electronic, and magnetic properties as a function of thiol coverage
}

\author{
R. Cuadrado, J. M. Puerta, F. Soria, and J. I. Cerdáa) \\ Instituto de Ciencia de Materiales de Madrid, CSIC, Madrid 28049, Spain
}

(Received 17 April 2013; accepted 27 June 2013; published online 19 July 2013)

\begin{abstract}
We have studied the stability of thiolated $\mathrm{Au}_{38}$ nanoparticles (NPs) via density functional theory based calculations varying the coverage from 0 up to 32 molecules. Three different initial core arrangements were considered for the cluster, spherical, tubular, and bi-icosahedral, while thiol groups were attached to the cluster via the sulfur atom either as single molecules or forming more complex staple motifs. After molecular dynamics runs several metastable configurations are found at each coverage thus allowing to analyze the properties of the NPs in the form of ensemble averages. In particular, we address the structural and electronic properties as a function of the number of thiols. The study emphasizes the strong influence of the core structure on the stability of the NPs, and its interplay with the thiol coverage and adsorption geometries. The magnetic properties of the NPs have also been explored via spin-polarized calculations including spin-orbit coupling. No evidence for the existence of a robust intrinsic ferromagnetism is found in any of the structures. (C) 2013 AIP Publishing LLC. [http://dx.doi.org/10.1063/1.4813615]
\end{abstract}

\section{INTRODUCTION}

Thiolate protected gold nanoparticles (NPs) have become a field of active research in the last decade. Gold NPs are not only interesting for their optical, ${ }^{1}$ biomedical, ${ }^{2,3}$ and technological ${ }^{1}$ applications, but they also constitute challenging systems from the fundamental point of view. ${ }^{4}$ Despite the existence of a wealth of works both on bare and molecule capped Au clusters, ${ }^{5-17}$ the ultimate structure of most of these NPs still remains unclear.

The adsorption geometry of the thiols both on gold NPs and in the form of self-assembled monolayers (SAMs) on the $\mathrm{Au}(111)$ surface has been subject of intense debate. A major breakthrough was provided by Maksymovych et al. ${ }^{7}$ who, based on Scanning Tunneling Microsocpy (STM) images and total energy calculations, challenged all previous adsorption models for thiolate SAMs on the $\mathrm{Au}(111)$ surface proposing a RS-Au-RS motif where a gold atom lifted from the herringbone reconstruction binds to two sulfurs which are themselves adsorbed at top positions. This adsorption structure, denoted as the staple motif, was immediately confirmed for gold NPs as well. Jadzinsky et al. ${ }^{5}$ reported the crystallization and X-ray structure determination of a $p$-mercaptobenzoic acid ( $p$-MBA)-protected gold NP comprising 102 gold atoms and $44 p$-MBAs. They found that its atomic structure consists of an approximately $D_{5 h}$-symmetric $\mathrm{Au}_{79}$ metallic core with a protective gold-thiolate layer of composition $\mathrm{Au}_{23}(p-$ MBA $)_{44}$ arranged as single and triple staple motifs: $\mathrm{Au}(\mathrm{RS})_{2}$ and $\mathrm{Au}_{2}(\mathrm{RS})_{3}$. More recently, Zhu et al. ${ }^{6}$ crystallized $\mathrm{Au}_{25}$ NPs and found a mixture of single and double staples embedding a $\mathrm{Au}_{13}$ core with $D_{2 h}$ symmetry. The structure of such

\footnotetext{
a) Author to whom correspondence should be addressed. Electronic mail: jcerda@icmm.csic.es
}

highly stable NPs, among others, has been rationalized under the superatom concept, which assumes a closed shell system for the core with all the NPs chemical activity confined to the thiolate-gold layer. ${ }^{18,19}$

From the theoretical point of view, and until the work of Maksymovych et al., ${ }^{7}$ density functional theory (DFT) based calculations for a thiolate covered $\mathrm{Au}(111)$ surface mainly favored bridge adsorption for the sulfur, with 3fold adsorption sites becoming slightly more stable only at high coverages. Regarding NPs, most works have focused on the computationally accessible $\mathrm{Au}_{38}$ cluster covered by methanethiolates $\left(\mathrm{R}=\mathrm{CH}_{3}\right)$. In the early work of Häkkinen et al. ${ }^{8}$ for the $\mathrm{Au}_{38}\left(\mathrm{CH}_{3} \mathrm{~S}\right)_{24}$ system, the sulfurs were found to adsorb at bridge sites covering a truncatedoctahedral face-centered-cubic $(f c c)$ spherical core. However, such standard model has been successively improved either via the replacement of bridge adsorption by staple motifs - single $^{9}$ or double ${ }^{10}$ or as ring-like tetraunits ${ }^{11}$ —and by exploring different core reconstructions. ${ }^{12,14}$ The most stable structure reported to date consists of a bi-icosahedral $\mathrm{Au}_{23}$ core covered by nine-both single and double-staples: $[\mathrm{Au}]_{5+18}\left[\mathrm{Au}\left(\mathrm{CH}_{3} \mathrm{~S}\right)_{2}\right]_{3}\left[\mathrm{Au}_{2}\left(\mathrm{CH}_{3} \mathrm{~S}\right)_{3}\right]_{6} \cdot{ }^{12}$

However, and despite some degree of tuning on the composition, size, and electronic properties of Au NPs can be achieved by controlling the balance between the size and the nature of the capping molecules, ${ }^{20}$ single crystals for these clusters have only been obtained for very few selected cases. Therefore, most of the properties measured for these clusters do not correspond to a unique highly stable structure but represent an average over a distribution of metastable phases where the thiolate coverage, the size and configuration of the core, and the structure of the capping layer are expected to change from NP to NP. In this line, Jiang et al. ${ }^{9}$ performed a DFT-based molecular dynamics (MD) study for $\mathrm{Au}_{38}\left(\mathrm{CH}_{3} \mathrm{~S}\right)_{x} \mathrm{NPs}$, varying $x$ from 2 to 
24 methylthiolate. They found that the staple mofits represented a key factor in the NP stability with respect to bridge adsorption inducing as well a drastic restructuring of the gold inner core. Furthermore, at large thiol coverages staple dimerization- $\mathrm{Au}_{2}\left(\mathrm{CH}_{3} \mathrm{~S}\right)_{3}$ - showed up in the optimized geometries. ${ }^{9}$

On the other hand, the magnetic character of gold NPs is probably their most intriguing property, ${ }^{21}$ as noble metals are diamagnetic in their bulk phases. In the last years several experimental groups have reported the appearance of a magnetic polarization in ligand-coated gold $\mathrm{NPs}^{22-27}$ mainly based on superconducting quantum interference device (SQUID) or X-ray magnetic circular dichroism (XMCD) measurements. Still, there is a wealth of contradictory experimental results about the size of the magnetic moments, their origin or the ferromagnetic character of the particles. ${ }^{21} \mathrm{Sev}-$ eral different theoretical explanations have been posed ${ }^{22,28-33}$ although a general consensus is still far from being reached. DFT based studies for thiol protected Au NPs ${ }^{29,32}$ can indeed account for the appearance of a net magnetic moment, although the gold clusters considered were small with only one thiol group attached. Still, the explanation for the origin of the ferromagnetism differs among the two works. Whereas González et al. ascribe it to a local spin-symmetry breaking process resulting from the interaction between the $S$ linker and the gold atom, ${ }^{29}$ Ayuela et al. ${ }^{32}$ encountered $1 \mu_{B}$ magnetic moments for clusters with an odd number of electrons arising from the singly occupied molecular orbital (SOMO) which is of $s p$ character but, surprisingly, has little weight along the S-Au bonds. Alternative phenomenological mechanisms have been proposed as well, such as the creation of $e$ holes in the $5 d$ shell of the gold atom upon bonding to the sulfur, ${ }^{22}$ or the Fermi hole effect whereby an uncompensated spin polarization develops around this hole which is located at the surface, ${ }^{28}$ or giant surface orbital momenta which, via spin-orbit interactions, pin the magnetic moment along the surface normal ${ }^{30}$ or, most recently, an orbital origin involving conduction electrons which are driven into persistent currents even in the absence of an external magnetic field. $^{33}$

In this work we first perform a structural study similar to that of Jiang et al. ${ }^{9}$ for the thiol protected $\mathrm{Au}_{38}$ cluster but exploring an ampler set of phases covering thiol coverages up to 32 molecules per NP and different core reconstructions. Furthermore, a detailed analysis is presented for both the structural and electronic properties of these NPs with the aim of determining the most important factors in their stabilization. Finally, we address any magnetic properties after spin polarized calculations including the spin-orbit coupling (SOC) within the DFT formalism. ${ }^{34}$ The paper is organized as follows. In Sec. II we will describe the details of the DFT based calculations. In Sec. III we present the trial structural models analyzed, and next the results of the simulations, first focusing on the structural and total energies of the different models, next analyzing their electronic structure, and finally their magnetic properties, always paying attention to the thiol coverage dependence. Finally, Sec. IV is devoted to a final discussion and the conclusions derived from this work.

\section{THEORY}

We assume throughout this work $\mathrm{Au}_{38}\left(\mathrm{C}_{2} \mathrm{H}_{5} \mathrm{~S}\right)_{N_{t h}} \mathrm{NPs}$, with $N_{t h}$ ranging from 0 to 32 , so that the coverage is varied from $\theta_{t h}=0$ to $\theta_{t h} \approx 100 \%$. The choice of an ethanethiol $\left(\mathrm{R}=\mathrm{C}_{2} \mathrm{H}_{5}\right)$, at contrast with previous studies which assume a methanethiol $\left(\mathrm{R}=\mathrm{CH}_{3}\right)$, was made to ensure that the chemical environment for the sulfurs in longer chains is accurately reproduced.

All calculations have been performed with the ab initio DFT based SIESTA code. ${ }^{35}$ We employed the Local Density Approximation (LDA) for the exchange and correlation (XC) energy based on Ceperley and Adler's parametrization. ${ }^{36}$ The interaction of the core electrons with the valence electrons is replaced by non-local and norm-conserving pseudopotentials based on the Troullier-Martins scheme. ${ }^{37}$ We generated such pseudopotentials for all species of interest, namely: Au, $\mathrm{S}, \mathrm{C}$, and $\mathrm{H}$, always under LDA. In order to reduce the computational cost no core corrections for the $\mathrm{XC}$ were included in the pseudopotentials in the geometrical optimizations. The AO basis set employed to describe the valence states is calculated by solving the Schrödinger equation of the bare atoms under a confinement potential, so that the solutions become strictly zero beyond a certain cut-off radius. The spatial range of each $\mathrm{AO}$ is set by imposing a $100 \mathrm{meV}$ energy cost due to the confinement potential. We used the so-called Double Zeta Polarized (DZP) scheme, whereby occupied shells in the bare atom are described by double- $\zeta$ orbitals while the AOs for an empty shell with angular momentum $l$ are obtained after polarizing the $l-1$ shell. Three-center integrals were computed over a 3D mesh in real space using as cutoff parameter 300 Ry (equivalent to a $0.1 \AA^{3}$ resolution) while, unless otherwise stated, the temperature employed in the Fermi-Dirac distribution (smearing) was set to $50 \mathrm{meV}$. This calculation parameter set yielded an overall numerical accuracy on the calculated energy differences of the order of $10 \mathrm{meV}$.

The choice of LDA instead of the generalized gradient approximation (GGA) ${ }^{38}$ was motivated by the fact that the former reproduced more accurately the experimental bulk $\mathrm{Au}$ $f c c$ lattice parameter $\left(a_{L D A}=4.08 \AA, a_{G G A}=4.19 \AA\right.$, and $a_{\text {exp }}$ $=4.08 \AA$ ). Nevertheless, we also carried out several tens of calculations under GGA in order to to check the influence of the $\mathrm{XC}$ on the calculated properties. Although GGA yields smaller binding energies, the overall trends with thiol coverage were found qualitatively very similar to the LDA results. Other sources of inaccuracies are the neglect of van der Waals (vdW) forces and the well-known basis set superposition error (BSSE) associated to the use of a localized basis set. However, we have checked for some selected cases that their inclusion in the evaluation of the adsorption energies via semi-empirical pair-potentials $\mathrm{s}^{39,40}$ for the former and counter poise corrections (CP) for the later ${ }^{41}$ do not change the conclusions of this work.

At each thiol coverage there exist many different metastable phases depending on the core reconstruction, the adsorption sites as well as on how close from each other are thiols placed. In order to explore in some detail such a complex total energy landscape we have performed MD runs for different initial NP structures—see Subsection III A—starting 

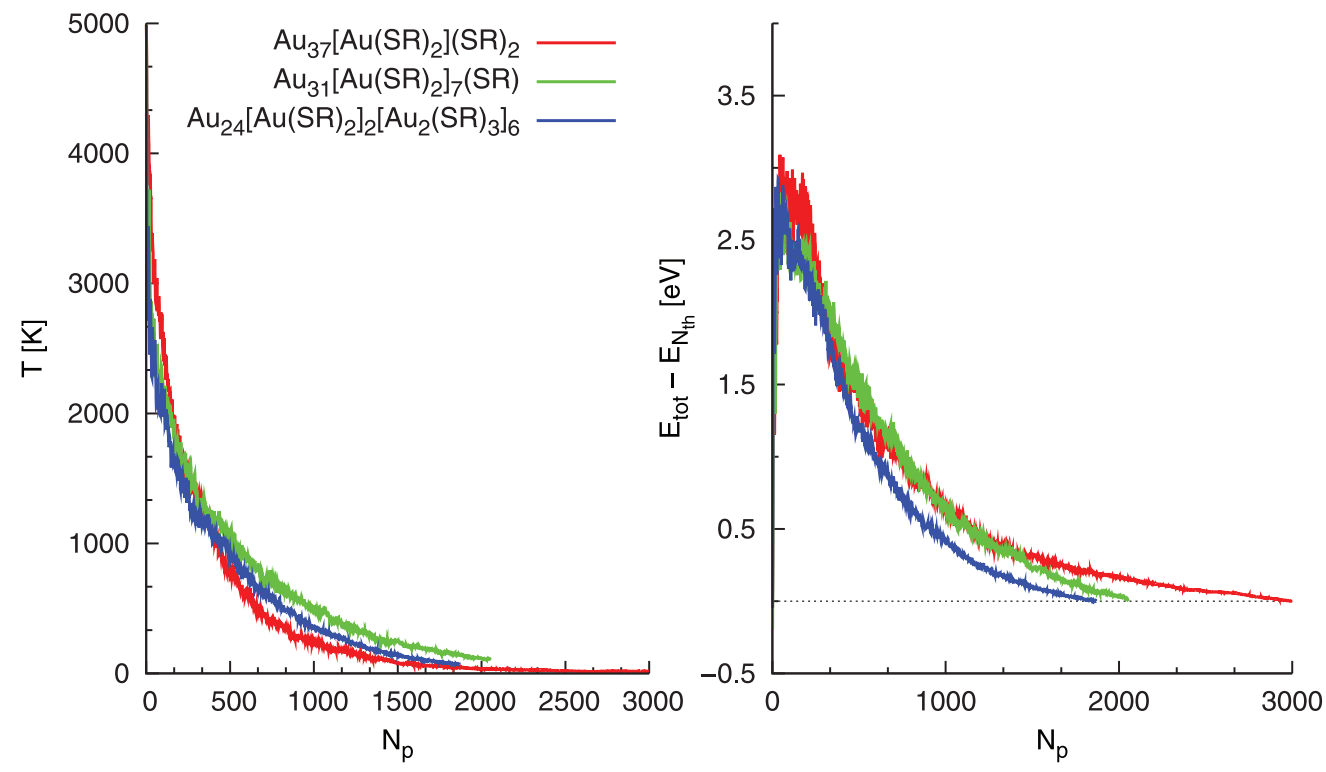

FIG. 1. Evolution of the ionic temperature (left) and total energy (right) of the system at each step in the SA runs for three different NPs,

at an initial large temperature, $T_{I}$, and next slowly cooling the system. Here we set $T_{I}=5000 \mathrm{~K}$ in order to allow the thiol migration to nearby sites, but preventing thiol dehydrogenation, which routinely occurred at around $7000 \mathrm{~K}$. The time step was set to 1 fs for all cases, while as the coverage increased the number of MD steps was increased accordingly. In Figure 1 we present the evolution of the system's temperature and adsorption energy (see below) during a MD run for three different NPs. In general, the MD runs were stopped when the temperature was around $50 \mathrm{~K}$, since no sensible atomic movements were found below this temperature. The resulting structures were next refined by conjugate gradient minimizations until forces on the atoms were smaller than $0.05 \mathrm{eV} / \AA$.

The $N_{t h}$ thiols adsorption energy per NP is calculated according to

$$
E_{i, N_{t h}}^{a d s}=N_{t h} E_{t h}^{g}+E^{\mathrm{Au}}-E_{i, N_{t h}}
$$

where $E_{t h}^{g}$ is the total energy for a thiol in the gas phase, $E^{A u}$ that of the bare cluster and $E_{i, N_{t h}}$ that of the Au NP capped with $N_{t h}$ thiols for a given metastable phase, $i . E_{N_{t h}}^{a d s}$ is the sum of two competing contributions: the interaction energy between the thiols and the core atoms and the energy cost associated to the core restructuring. We calculated $E^{\mathrm{Au}}$ by optimizing the $f c c$ spherical cluster ${ }^{15}$ and we used this value for all models and coverages.

Given that each MD run yielded a different metastable structure and that despite the annealing scheme one cannot be certain of having reached the most stable geometry, ${ }^{17}$ we have found appropriate to present our results in the form of statistical averages over the various phases found at each coverage, taking care of their relative stabilities. For instance, the ensemble average adsorption energy at each $N_{t h}$ value is given by

$$
\left\langle E_{N_{t h}}^{a d s}\right\rangle=\frac{\sum_{i=1}^{M_{t h}} E_{i, N_{t h}}^{a d s} e^{\left(E_{N_{t h}}^{\max }-E_{i, N_{t h}}\right) / k T}}{\sum_{i=1}^{M_{t h}} e^{\left(E_{N_{t h}}^{\max }-E_{i, N_{t h}}\right) / k T}},
$$

where $M_{t h}$ denotes the number of relaxed configurations calculated at each thiol coverage, $E_{i, N_{t h}}^{a d s}$ its corresponding adsorption energy, and $E_{N_{t h}}^{\max }$ the largest adsorption energy among the ensemble of structures for a given $N_{t h}$. The fictitious Boltzmann factor was assigned a large value, $k T=1 \mathrm{eV}$, in order to increase the probability of occurrence of less stable structures. Analogous averages have been performed for other quantities such as interatomic distances, Mulliken populations, energy gaps, or magnetic moments. We feel that our statistical approach is more meaningful when comparing the theoretical results against experimental data than considering instead the most stable structures at each coverage.

We also examined the fundamental gap of the NPs, $E_{i, N_{t h}}^{F G}$, defined as the energy required to create an unbound electronhole pair: ${ }^{42}$

$$
E_{i, N_{t h}}^{F G}=E_{i, N_{t h}^{+}}+E_{i, N_{t h}^{-}}-2 E_{i, N_{t h}}=I_{i, N_{t h}}-A_{i, N_{t h}},
$$

where $I_{i, N_{t h}}$ and $A_{i, N_{t h}}$ are the ionization energy and electron affinity of NP $i$, respectively, and $E_{i, N_{t h}^{ \pm}}$is the total energy of the system after removing or adding one $e$ (the artificial monopole-monopole interactions appearing in such charged systems were corrected via appropriate Madelung sums). Equation (2), generally denoted as the $\triangle \mathrm{SCF}$ method, represents a reasonable approximation to the gap in isolated systems as reflected in Table I, where it has been tested for the individual atoms. Compared against the LDA derived gaps $E_{G}$

TABLE I. LDA derived gaps $E_{G}$, fundamental gaps $E_{F G}$, and experimental values $I-A$ for the isolated atoms given in the first column. All energies in eV.

\begin{tabular}{lrrr}
\hline \hline & $E_{G}$ & $E_{F G}$ & $I-A$ \\
\hline $\mathrm{H}$ & 14.1 & 11.4 & 12.8 \\
$\mathrm{C}$ & 8.2 & 11.2 & 10.0 \\
$\mathrm{~S}$ & 13.5 & 9.8 & 8.3 \\
$\mathrm{Au}$ & 0.6 & 7.8 & 6.9 \\
\hline \hline
\end{tabular}


$=\epsilon_{\text {LUMO }}-\epsilon_{\text {HOMO }}$, a better agreement with the experimental values is found in all cases, particularly for the Au atom.

The magnetic properties of all the relaxed NPs are finally investigated within the framework of the Local Spin Density (LSD) approximation. Given the large Au mass, we included SOC via the use of fully relativistic pseudopotentials (FR-PPs) as recently implemented within the GREENSIESTA package. ${ }^{34,43,44}$ The FR-PP approach is highly efficient compared to full potential formalisms yet it remains sufficiently accurate when determining spin-orbit splittings or Magnetic Ansisotropy Energies (MAEs) ${ }^{34}$ Nevertheless, the FR-PP formalism requires the inclusion of partial core corrections $^{45}$ in the evaluation of the XC potential ${ }^{34}$ and this, in turn, demands finer real space meshes for a proper convergence of the magnetic properties. Here, we increased the mesh cut-off to 700 Ry (or $0.06 \AA^{3}$ resolution) when computing the magnetic moments of all the NPs metastable phases obtained in the previous stage.

\section{RESULTS AND DISCUSSION}

\section{A. Structural models}

We have considered three different reference core configurations for the $\mathrm{Au}_{38}\left(\mathrm{C}_{2} \mathrm{H}_{5} \mathrm{~S}\right)_{N_{t h}} \mathrm{NPs}$. The first one is based on the most commonly assumed truncated-octahedral $f c c \mathrm{Au}_{38}$ clean cluster, which is depicted in Fig. 2(a), and will be denoted hereafter as the spherical cluster. In the course of the study we identified a novel Au core arrangement which presented a high stability at medium coverages. This $\mathrm{Au}_{21}$ cluster will be denoted as tubular and is depicted in Fig. 2(b). It consists of four planar structures with a local hexagonal symmetry, each formed by a Au atom at the center surrounded by six neighbors. The planar structures are stacked following a $h c p$ sequence. The third core configuration is the bi-icosahedral $\mathrm{Au}_{23}$ cluster introduced by Jiang ${ }^{9}$ - depicted in Fig. 2(c) and which will be denoted by bi-icos. Note, however, that recent studies on clean $\mathrm{Au}_{38}$ NPs have revealed more stable geometries which are either amorphous ${ }^{14}$ or tetrahedral-like. ${ }^{16}$ Employing such optimum geometries may have some impact on the thiol adsorption geometries and energies, although we feel the main trends derived in the present work should remain valid given the large restructuring of the NP as soon as a few ligands are attached — see below. Yet, further simulations, beyond the scope of the current work, should be addressed in order to confirm this fact.

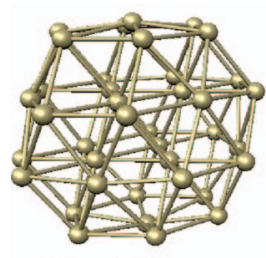

(a) Spherical

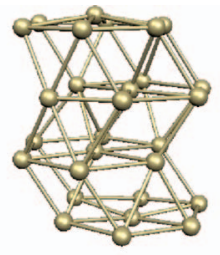

(b) Tubular

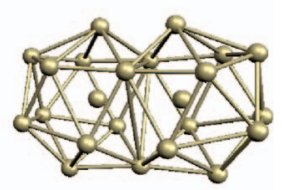

(c) Icosahedral
FIG. 2. Side views of the reference configurations for the Au cores employed in this work. (a) Spherical $\mathrm{Au}_{38}$, (b) tubular $\mathrm{Au}_{28}$ and, (c) bi-icos $\mathrm{Au}_{23}$ structures.
Given these three reference core models, we generated initial trial structures for all thiol coverages according to the following criteria:

- spherical: for each coverage, we always placed the corresponding number of thiols at bridge sites exposed at the surface of the cluster. In a first set of MD runs we distributed the molecules randomly around the cluster, while in a second set of runs we increased the number of adjacent thiols on the surface thus favoring the creation of staple motifs.

- bi-icos: here we covered the bi-icosahedral cluster by 9 staple motifs following the structure of Pei et al. ${ }^{12}$ Initial guesses for all other lower coverages were generated by simply removing the appropiate number of thiols while retaining the $\mathrm{Au}$ atoms in the staples. In the MD simulations, the latter immediately attached to the cluster inducing further restructuring.

- tubular: starting from the $N_{t h}=19$ case, for which we found a particularly stable structure after protecting the tubular Au core by eight staples (see below) we added or removed thiols following an analogous scheme as for the bi-icos case.

Furthermore, since we often found strong variations in the thiol adsorption energy between consecutive coverages and for the same reference Au core, we performed additional MD runs for the less stable cases using as starting structures the more stable ones obtained for the previous or next coverage after adding or removing one thiol group. This way we were able to improve the stability significantly in most cases. Let us finally note that alternative model approaches such as adding RS-Au-RS staples to the NP could lead new structures with a high stability but different number of Au atoms. However, we have preferred to reduce the number of degrees of freedom ${ }^{17}$ by fixing the number of gold atoms in all structures, despite this is a rather stringent condition.

\section{B. Total energies and structural properties}

Figure 3 summarizes the stability of the different NPs studied in this work as a function of the thiol coverage. In Fig. 3(a) we plot the mean adsorption energy per thiol, $\left\langle E_{N_{t h}}^{a d s}\right\rangle / N_{t h}$. The individual values for each NP are plotted as red, green, and blue symbols to designate NPs which initial core structures were spherical, tubular, and bi-icos, respectively. Furthermore, we employ circles for those NPs where staple motifs are predominant and crosses for those where we also find a considerable amount of sulfur atoms adsorbed at bridge sites. The graph shows an overall decrease of the adsorption energy with increasing number of thiols or, in other words, the NPs capability of adsorbing thiols decreases with coverage. In accordance with Jiang et al. ${ }^{9}$ we find an abrupt decrease in the mean adsorption energy for $N_{t h}<5$, while beyond this coverage the dependence is weaker, attaining values from $4.0 \mathrm{eV}$ down to $3.3 \mathrm{eV}$. Notice that our adsorption energies are significantly larger than those reported by Jiang et $a l .{ }^{9}(2.3-3.4 \mathrm{eV})$ or by Maksymovych et al $^{7}$ for adsorption on the $\mathrm{Au}(111)$ gold surface $(\sim 2 \mathrm{eV})$. The difference 


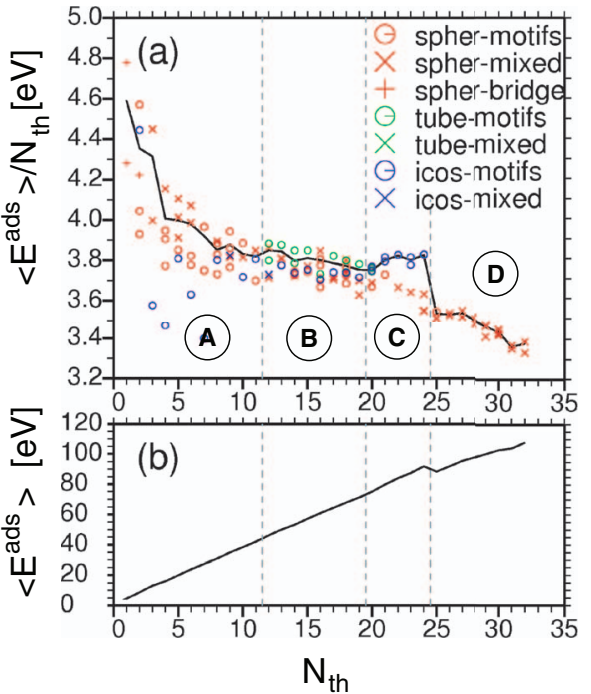

FIG. 3. (a) Adsorption energy per thiol, $E_{N_{t h}}^{a d s} / N_{t h}$. Thick solid line refers to the mean value, $\left\langle E_{N_{t h}}^{a d s}\right\rangle / N_{t h}$, while symbols provide the individual energies for all NPs studied - see legend. Vertical dashed lines define the four regions, $\mathrm{A}, \mathrm{B}, \mathrm{C}$, and D discussed in the text. (b) Mean adsorption energy, $\left\langle E_{N_{t h}}^{a d s}\right\rangle$, as a function of the number of thiols, $N_{t h}$.

should be ascribed mainly to the LDA employed in our work and, to a lesser extent, to the replacement of methanethiols by ethanethiols. Indeed, after relaxing up to 54 structures under GGA we found that $E_{N_{t h}}^{a d s} / N_{t h}$ decreased in all cases by 0.7 $\pm 0.1 \mathrm{eV}$ while the evolution of the energies with coverage remained unaltered. Additionally, LDA relaxations with ehtyl instead of methyl groups consistently yielded adsorption energies around $0.2 \mathrm{eV}$ larger without modifying the trend shown in the figure.

After inspecting the initial core configuration of the best structures at each coverage we have defined four different regions in the graph, $A-D$, separated in the figure by the vertical lines. Region $A$ corresponds to small coverages, $N_{t h} \leq 11$, where the most stable structures arise from the spherical core and end up, in general, with a mixture of staples and bridge adsorbed thiols - red crosses. Only at the very lowest cover- ages we obtained configurations with only bridge adsorption. Recall that structures solely covered by motifs need not correspond to the most stable ones-circles appear in this region above crosses only for $N_{t h}=2,7$, and 10 . This implies that staple formation is not the unique stabilization mechanism, but the core restructuring and the relative positions of the sulfurs are relevant competing factors. As representative cases, we plot in Figs. 4(a)-4(d) four different relaxed geometries within region $A$ consisting of: (a) two bridge adsorbed thiols, clearly less stable than those forming motifs, $(b, c)$ mixture of a bridge adsorbed thiol plus one or two staples and, (d) the less stable structure at $N_{t h}=7$ consisting of two single staples and a double one. The degree of the core restructuring is patent as the number of thiols is increased.

At intermediate coverages, $12 \leq N_{t h} \leq 19$, the most stable NPs correspond to the novel tubular core geometry depicted in Fig. 2(b)-green symbols within region $B$. The core is predominantly protected by staple motifs. As shown in Figs. 4(e) and 4(f) for the $N_{t h}=19$ case, the thiols adsorb along the side of the tube forming up to six single staples and two double ones linking alternative planes with an additional thiol bound at a bridge site in the lower plane. The rest of tubular geometries at other coverages presented similar features.

Region $C$ comprises the range $20 \leq N_{t h} \leq 24$, where we find that the bi-icos $\mathrm{Au}_{23}$ core covered by staples is clearly the most favourable model-blue circles. The remarkable stability of this structure is reflected in an increase in the thiol adsorption energy at contrast with the general trend of smaller $\left\langle E^{a d s}\right\rangle / N_{t h}$ values with increasing $N_{t h}$. We depict in Figs. 4(g) and 4(h) two such structures for $N_{t h}=22$ and 24, respectively, the latter corresponding to the model of Pei et al. ${ }^{12}$ Here all surface gold atoms are protected by a thiol group whereas in the tubular configurations we always find a small fraction of unsaturated surface atoms. Recall, however, that the level of core restructuring from the spherical arrangement to the bi-icos one is much higher than to the tubular one, implying that in the former case the associated energy barriers should be substantially larger. Indeed, none of the MD runs starting with an spherical core ended up in the bi-icos structure.

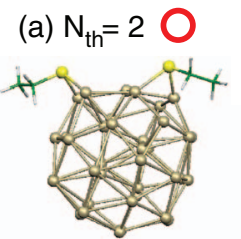

(b) $\mathrm{N}_{\mathrm{th}}=4 \mathrm{X}$

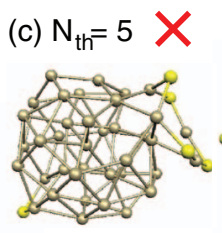

(d) $\mathrm{N}_{\mathrm{th}}=7 \mathrm{O}$

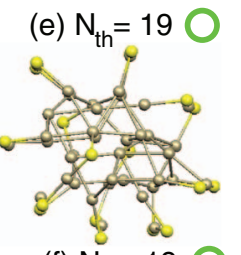

(f) $\mathrm{N}_{\text {th }}=19$
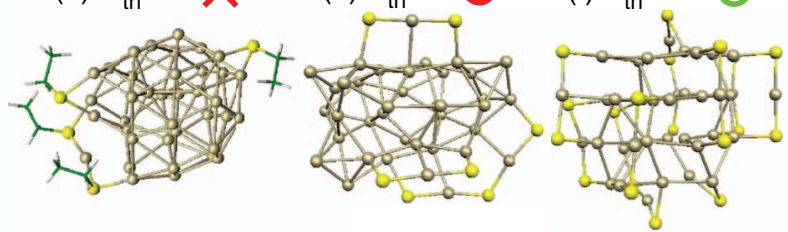

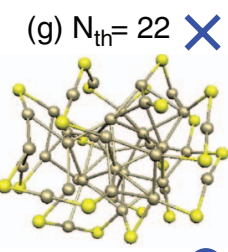

(h) $\mathrm{N}_{\text {th }}=24 \mathrm{O}$

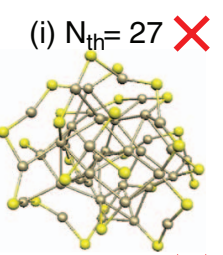

(j) $\mathrm{N}_{\mathrm{th}}=30 \mathrm{X}$
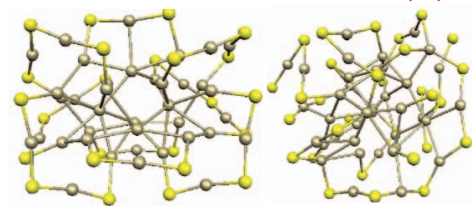

FIG. 4. (a)-(j) Relaxed atomic configurations for several NPs with different thiol coverages obtained in this work. Thiol groups have been removed for clarity, only showing the S (yellow) and Au (golden) atoms. The number of thiols per NP is indicated at the top of each figure, while the large symbol to its right (crosses or circles) are used to classify the type of structure-see legend in Fig. 3. Recall that the NPs depicted need not be the most stable ones at each coverage. (a-d) The low coverage region $A$ in Fig. 3, (e,f) top and side view of the most stable structure at $N_{t h}=19$, corresponding to a tubular inner structure decorated with staples. $(\mathrm{g}, \mathrm{h})$ Two most stable configurations within region $C$, both obtained from an initial bi-icos core structure. (i,j) The high coverage region, $D$. 
For the largest thiol coverages, $N_{t h}>24$ or region $D$, we only considered an initial spherical core. The resulting adsorption energies show a sudden drop with respect to the bi-icos cases and basically continue the trend of the spherical model at smaller coverages-red symbols. Nevertheless, the energy gain upon further thiol adsorption is still considerable. Two relaxed structures within this region are presented in Figs. 4(i) and 4(j). They are densely decorated with motifs, which may appear either as single or double staples or even larger $\mathrm{Au}_{3}(\mathrm{RS})_{4}$ units, while individual thiols adsorbed at top sites or unsaturated (non-cyclic) polymeric ...-Au-RS-Au-RS chains are also found. The large amount of staples present induces an enormous reconstruction of the core, which hardly resembles any more its initial $f c c$ arrangement.

A more general picture of the NPs stability is obtained from the mean adsorption energy, $\left\langle E_{N_{t h}}^{a d s}\right\rangle$, presented in Fig. 3(b) -individual energies are not shown since, given the large energy range, the data points collapse into the solid line. We observe an almost linear increase with the number of thiols throughout the entire range. No saturation is found even at the largest coverages, suggesting that thiols can be adsorbed until a $[\mathrm{Au}(\mathrm{RS})]_{38}$ cyclic chain (polymer model) is formed. Only in region $C$ one finds a slight increase in the slope arising from the larger stability of the bi-icos model, while beyond 24 thiol coverage the slope returns to its initial value. As a result, a local maximum at $N_{t h}=24$ is found precisely for the model of Pei et al., ${ }^{12}$ although this structure corresponds to a metastable phase since the NP stabilities for $N_{t h}$ $\geq 27$ are clearly larger. Figure 3(b) shows, on the one hand, the remarkable capability of the $\mathrm{Au}_{38}$ cluster to uptake thiols and, on the other hand, the bi-icos model should be hard to achieve experimentally since adding just three more thiols to the NP renders it more stable without having to overcome the large energy barriers associated to such a drastic core reconstruction.

Figure 5 shows the distribution of $\mathrm{Au}-\mathrm{Au}$ distances among the core atoms in the NP for the different thiol coverages. We find an overall increase in the mean nearest neighbor distances, $\left\langle d_{\mathrm{Au}-\mathrm{Au}}\right\rangle$, with increasing coverage up to around $2 \%$, ranging from $2.80 \AA$ for the clean spherical core to $2.86 \AA$ at high coverages. Region $\mathrm{C}$ deviates slightly from this general behavior with the bi-icos models presenting even larger $\mathrm{Au}-\mathrm{Au}$ distances $(2.90 \AA$ or $4 \%)$ as indicated by the dashed blue line in the figure. This expansion is in line with (albeit smaller than) that found from EXAFS experiments by Crespo et al. ${ }^{22}$ who reported a value of $5 \%$ for $d_{\mathrm{Au}-\mathrm{Au}}$ (from $2.83 \AA$ to $2.98 \AA$ ). The expansion may be attributed to the weakening of the $\mathrm{Au}-\mathrm{Au}$ bonds at the expense of the stronger $\mathrm{Au}-\mathrm{S}$ bonds.

\section{Electronic properties}

We first address the behavior of the Mulliken populations in order to identify any charge transfer mechanisms within the NP and follow their evolution with the thiol coverage. We have grouped the gold atoms into two sets: those forming the inner core region, $\mathrm{Au}_{\text {core }}$, and the rest, $\mathrm{Au}_{\text {surf }}$, irrespective of whether they are bonded to a sulfur or not. Although the distinction between the two types of atoms is somewhat

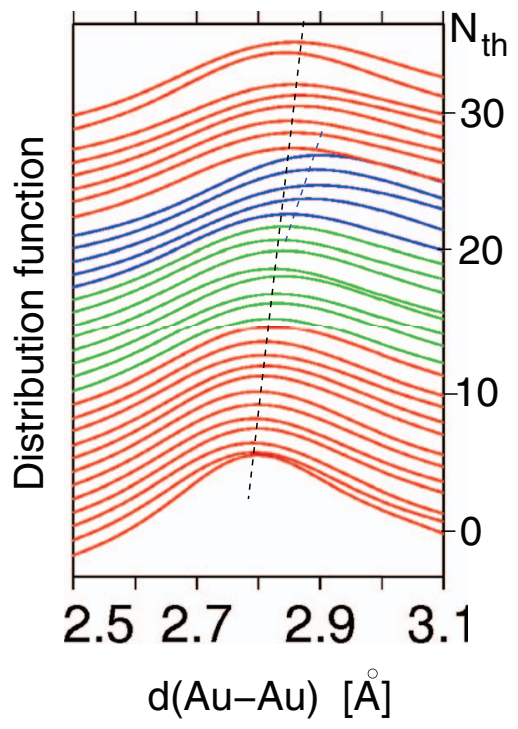

FIG. 5. Mean distribution function for the $\mathrm{Au}-\mathrm{Au}$ first nearest neighbour distances among the core atoms. Each curve corresponds to an average over all configurations at a given coverage, $N_{t h}$ (given in the right axis). We have used red, green, and blue lines for regions $A$ and $D$, region $B$ and region $C$, respectively_-see Fig. 3(a). The dashed line is drawn to guide the eye.

ambiguous for complex structures, we checked that the precise criterion followed hardly modifies the averaged Mulliken populations. In Figure 6 we plot the mean charge transfers, $\langle\delta Q\rangle$, for the $\mathrm{Au}_{\text {core }}$ and $\mathrm{Au}_{\text {surf }}$ atoms as well as for the thiols as a function of $N_{t h}$. We define $\delta Q$ as the difference between the Mulliken populations and the charge of the isolated gold atoms or thiols. Despite the value of $\delta Q$ depends on the selected basis set, the trends shown in the figure were found robust after several test calculations.

At small and moderate coverages, regions $A$ and $B$, one finds that the thiols remain fairly neutral. As regards the gold atoms, at the lowest coverages there is a significant charge transfer from the core to the surface atoms, thus leading to a NP positively charged in its inner region and negatively at the surface- - see the inset in Figure 6. However, this charge transfer diminishes rapidly with increasing coverage. Curiously, in the 8-17 $N_{t h}$ range the thiols present spiky features with peaks

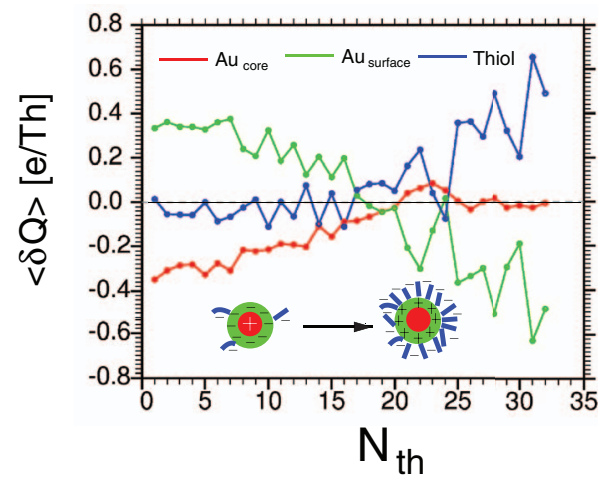

FIG. 6. Mean charge transfers as a function of the thiol coverage projected on the gold and surface atoms as well as on the thiols. The inset sketches the transition of the NPs charge distribution as the thiol coverage is increased from lower to higher coverages. 


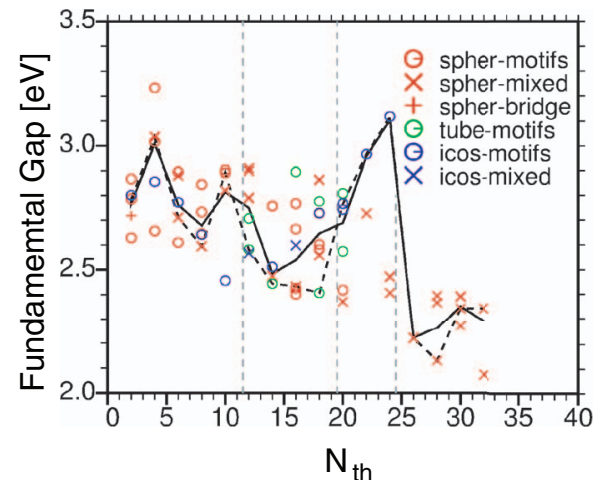

FIG. 7. Fundamental gaps for the $\mathrm{Au}_{38}\left(\mathrm{C}_{2} \mathrm{H}_{5}\right)_{N_{t h}}$ NPs considered in this work as a function of the coverage $N_{t h}$. Only those NPs with an even number of thiols are shown. Symbols follow the same scheme as in Fig. 3. Solid line gives the mean fundamental gap, while dashed that for the most stable structure at each coverage.

(dips) at odd (even) coverages which clearly anticorrelate with the surface gold charges.

Beyond $N_{t h}=20$, regions $C$ and $D$, the core atoms become neutral and all the charge compensation takes place between the thiols and the surface gold atoms, with increasing charge transfers from the latters to the formers as the coverage increases-see the inset. A notable exception is the $b i$ icos structure at $N_{t h}=24$, where we find hardly any charge transfers indicating that in this arrangement local dipole moments are minimized. Our low coverage results for the charge transfers are in accordance with the study of Ayuela et al., ${ }^{32}$ who found similar values for one thiol protected small gold clusters.

A routinely invoked argument to assess the stability of the NP structures is the value of the energy gap. We have calculated the fundamental gap, $E_{N_{t h}}^{F G}$, according to Eq. (3) for all NPs with an even number of electrons-equivalent to an even number of thiols-since those with an odd number are necessarily metallic if spin degeneracy is assumed-see Sec. III D. Their evolution with the thiol coverage is presented in Figure 7 following the same scheme as in Fig. 3. The gaps range between 2.0 and $3.3 \mathrm{eV}$, with the mean (solid line) showing a prominent peak for the bi-icos model at $N_{t h}=24\left(E^{F G}=3.1 \mathrm{eV}\right)$ and smaller peaks at $N_{t h}$
$=4(3.0 \mathrm{eV}), 10(2.8 \mathrm{eV})$, and $30(2.3 \mathrm{eV})$. Otherwise, there is a general tendency to smaller gaps as the coverage increases due to the proliferation of new states close to the Fermi level (see below). For the bi-icos model the largest gaps coincide with the most stable structures (dashed line) at coverages $N_{t h}$ $=22$ and 24 . However, this is by no means the general rule; in most cases, and specially at intermediate coverages, one finds small gaps $(\sim 2.5 \mathrm{eV})$ for the most stable structures. Therefore, the size of the fundamental gap cannot be regarded as a direct measure of the NPs stability.

Figure 8 displays the mean Projected Densities of States (PDOS) at selected coverages obtained after ensemble averaging. The projections are calculated onto the thiol molecules and onto the $\mathrm{Au}$ atoms grouped into three types: those belonging to the core $\left(\mathrm{Au}_{\text {core }}\right)$, those located at the surface and not bonded to any sulfur $\left(\mathrm{Au}_{\text {surf }}\right)$, and those bonded to at least a sulfur atom $\left(\mathrm{Au}_{S}\right)$. In all cases, increasing $N_{t h}$ tends to smear the highly peaked structure found for the isolated cluster and a hypothetical isolated thiol molecule (lower dark curves). This is particularly evident for the peaks at the Fermi level, which suffer a strong decrease already at $N_{t h}=1$.

The $d$-band, centered at $-4 \mathrm{eV}$, is the main feature in the Au PDOS with a width of around $5.5 \mathrm{eV}$. A peak at the lower band edge $(\sim-6 \mathrm{eV})$ of the $\mathrm{Au}_{\text {core }}$ develops at high coverages at the expense of the peak at the upper edge $(\sim-2 \mathrm{eV})$. The $\mathrm{Au}_{\text {core }}$ shows additionally a small characteristic peak at around $-9 \mathrm{eV}$ which is not seen for the rest of gold atoms. The surface Au PDOS suffer a narrowing of the $d$-band as expected from their reduced coordination. The effect is more pronounced for the $\mathrm{Au}_{S}$ mainly due to the covalent nature of the $\mathrm{Au}-\mathrm{S}$ bonds. The dominant peak at $\sim-2 \mathrm{eV}$ for $\mathrm{Au}_{\text {surf }}$ turns into a broad peak centered at $-3 \mathrm{eV}$ for $\mathrm{Au}_{s}$. For the special case of the bi-icos NP at $N_{t h}=24$ the spectra deviate somewhat from the general trend; the corresponding PDOS for the $\mathrm{Au}_{\text {surf }}$ presents several well defined peaks while the $-3 \mathrm{eV}$ one in the $\mathrm{Au}_{S}$ PDOS is enhanced and better resolved. As regards the thiols, there is a large shift of around $2 \mathrm{eV}$ towards lower energies for all the molecular orbital (MO) levels once bonded to the cluster. With increasing coverage the peaks broaden due to the hybridization and delocalization of the MOs. At the largest coverage of $N_{t h}=32$ a profusion of states appears below the Fermi level.
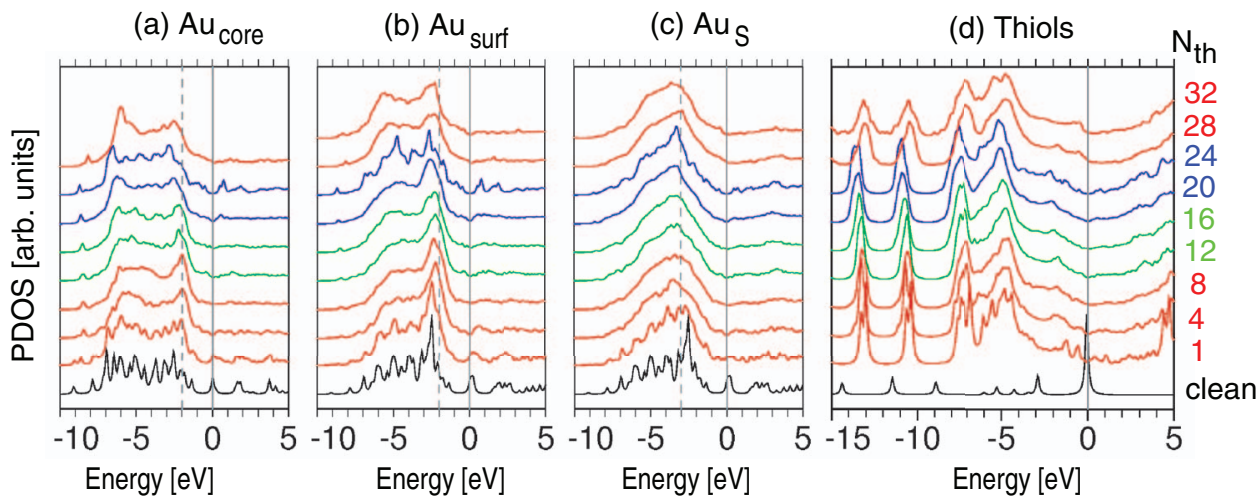

FIG. 8. Mean PDOS for different thiol coverages (given in the rightmost axis). The DOS are projected onto the (a) Au core atoms, (b) Au surface atoms, (c) Au atoms bonded to sulfur and, (d) thiol molecules. Dark lower spectra correspond to the isolated cluster (a-c) and the isolated thiol (d). The same color convention as in Fig. 5 has been employed. The energy origin is placed at the Fermi level (gray solid lines) while dashed gray lines are included to guide the eye. 


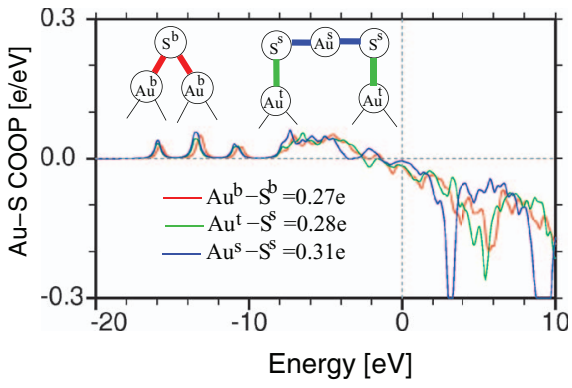

FIG. 9. Au-S COOPs for the different $\mathrm{Au}-\mathrm{S}$ bonds sketched in the insets. The curves have been calculated for two $\mathrm{Au}_{38}\left(\mathrm{C}_{2} \mathrm{H}_{5}\right)_{2} \mathrm{NPs}$, one with one single staple motif (blue and green lines) and the other with two Ss adsorbed at bridge sites (red). Numbers appearing in the legend correspond to the BOPs among the two atoms.

In order to gain further insight into the energy gain upon staple formation, we have compared the $\mathrm{S}-\mathrm{Au}$ bonding character between two $\mathrm{Au}_{38}\left(\mathrm{C}_{2} \mathrm{H}_{5}\right)_{2} \mathrm{NPs}$, one having a staple motif (most stable structure at this coverage) and a second one having two sulfurs adsorbed at bridge sites (less stable by $0.7 \mathrm{eV}$ ). Figure 9 presents the Crystal Orbital Overlap Populations (COOPs) for the three types of S-Au bonds sketched in the inset. Their respective Bond Order Populations (BOPs), given in the legend of the graph, are only slightly larger within the staple $\left(\mathrm{Au}^{S}-\mathrm{S}^{S}\right)$ than between the sulfur and the $\mathrm{Au}$ in the cluster (0.27-0.28e vs. 0.31e). The bonding character (positive COOPs) comprises the three energy peaks at low energieswhich mainly arise from the S- $s$ orbital-and the broadband between $-9 \mathrm{eV}$ and $-2 \mathrm{eV}$ - which involves the $\mathrm{S}-p$ and $\mathrm{Au}-$ $d$ states. Notice that this latter band is slightly narrower for the $\mathrm{Au}^{s}-\mathrm{S}^{s}$ COOPs (blue line) due to the contraction of the Au- $d$ states within the staple (see Fig. 8). An anti-bonding character (negative COOPs) develops at positive energies and also just below the Fermi level for the $\mathrm{Au}^{t}-\mathrm{S}^{s}$ and $\mathrm{Au}^{b}-\mathrm{S}^{b}$ cases. Nevertheless, the COOPs are very similar for the three types of bonds and, hence, cannot account for the large difference in stability among the two NPs, which may be attributed instead to the cluster reconstruction induced by the creation of the staple motif. We have checked that this general picture for the $\mathrm{Au}-\mathrm{S}$ bond remains at higher coverages.

\section{Magnetic structure}

Spin polarized calculations including SOC have been performed for all the relaxed metastable geometries described in Sec. III B considering different electronic temperatures, $k T$ $=0.1,5,10$, and $50 \mathrm{meV}$ in the Fermi-Dirac distribution function. The results are displayed in Figure 10 where we provide the mean magnetic moments (MMs) at each coverage and $k T$ as well as the individual moments for each NP (colored symbols). At the lowest temperature of $k T=0.1 \mathrm{meV}$ (dark solid line) we find MMs close to $1 \mu_{B}$ for all NPs with an odd number of electrons (or equivalently, with and odd number of thiols) while those NPs with an even number of electrons did not show any spin polarization except for a few cases discussed below. Inspection of the DOS at the Fermi level reveals that the magnetism originates in all cases from the exchange splitting of the SOMO. Hence, MMs of around $1 \mu_{B}$ for the odd

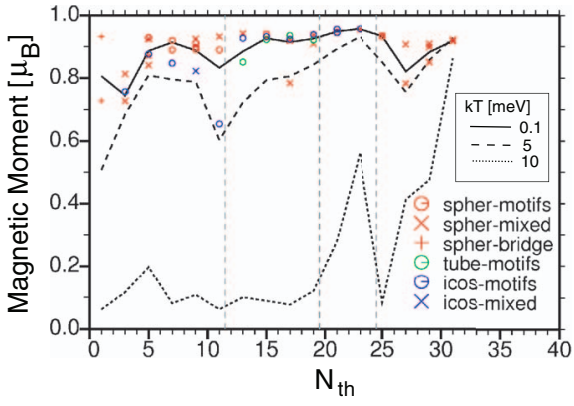

FIG. 10. Magnetic moments for those NPs with an odd number of thiols obtained for $k T=0.1 \mathrm{meV}$. Symbols follow the same scheme as in Fig. 3. Dark solid, dashed, and dotted lines correspond to the mean magnetic moments at $K T=0.1,5$, and $10 \mathrm{meV}$, respectively.

NPs is a trivial result which should always hold at sufficiently low temperatures regardless of the NP size. ${ }^{32}$ The relevant issue is the magnitude of the spin splitting which will determine the robustness of the NPs ferromagnetic character.

In Figures 11(a) and 11(b) we plot the spin resolved total DOS for two representative odd NPs with a net spin polarization. There is no clear shift in energy between the majority and minority spin DOS, while the SOC only induces small changes in the shape of the DOS mainly in the gold $d$-band (see dashed lines). The same DOS, but restricted to the vicinity of the Fermi level, are shown in (e,f). In both cases, and all others not shown, the splitting of the SOMO (dashed line) is small, attaining values below $50 \mathrm{meV}$. Spin density isosurfaces, $m(\vec{r})$, defined as the charge density difference between the majority and minority spins, are displayed in $(i, j)$, and they are basically the same as the charge density associated to the SOMO. The MMs tend to be localized at certain gold and sulfur atoms and are distributed around the entire molecule. As for the particles with an even number of electrons we have only found net MMs for the clean spherical cluster and for just two high coverage phases (region $D$ ). In figures $(\mathrm{c}, \mathrm{d})$, $(\mathrm{g}, \mathrm{h})$, and $(\mathrm{k}, \mathrm{l})$ we show their associated PDOS and spin densities. In these cases the frontier MOs are degenerate and split into several peaks; otherwise the picture is very similar to the odd electron cases with small SOC induced changes, small spin splittings and a high degree of delocalization in the spin density.

All these results may be cast under the well-known Stoner criterion which states that magnetism will develop in the NP if $\operatorname{DOS}\left(E_{f}\right)>1 / J$ where $J$ is the spin-spin coupling and $\operatorname{DOS}\left(E_{f}\right)$ is the density of states at the Fermi level (in our case, it corresponds to the height of the SOMO peak). Therefore, increasing $k T$ will smear this peak thus reducing $\operatorname{DOS}\left(E_{f}\right)$ until the MMs vanish. Indeed, if the spin-polarized calculations are performed at larger electronic temperatures (dashed and dotted lines in Fig. 10) the polarization clearly diminishes while at $k T=50 \mathrm{meV}$ all MMs vanish. Given the small $J$ coupling found in all cases (if any) we may regard the ferromagnetic character of the NPs as very weak and, certainly, cannot account for many of the experimental measurements which are already highly contradictory. ${ }^{21}$ On the other hand, total MMs of around $1 \mu_{B}$ results in an average atomic moment of around $0.026 \mu_{B}$ /atom, which is above that mea- 

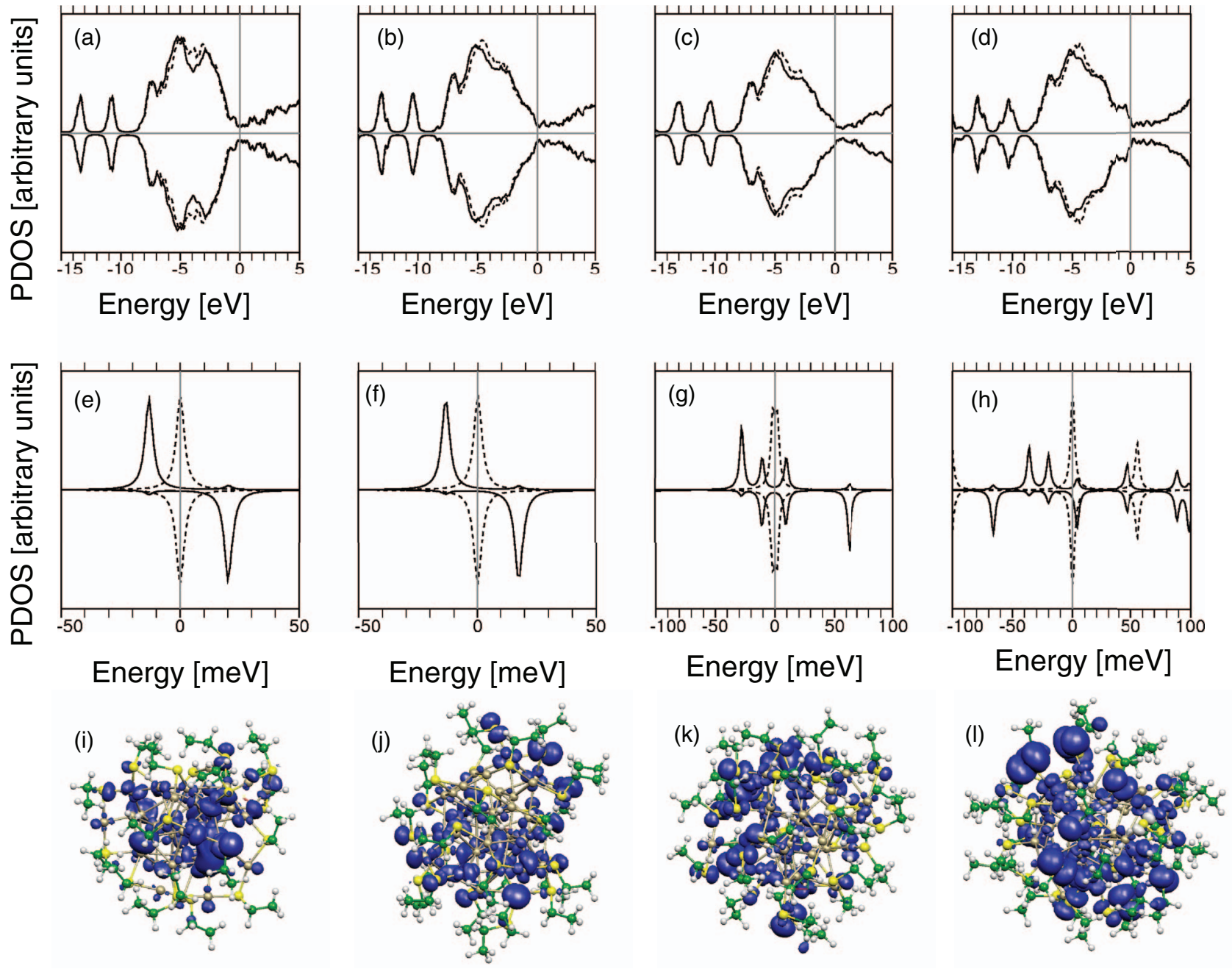

FIG. 11. (a)-(d) Total PDOS for NPs with $N_{t h}=17,25,28$, and 32, respectively, calculated without spin polarization (dashed lines) and with spin polarization and SOC (solid lines). Positive (negative) values for the majority (minority) spin components. (e-h) Same PDOS as in (a-d) but resolved around the vicinity of the Fermi level. (i-1) Isosurfaces of the net spin densities are superimposed on each NP.

sured in XMCD experiments (although they could be reconciled if one assumes that one out of every ten NPs is polarized) but substantially smaller than the large polarizations reported from SQUID magnetometry. ${ }^{21}$

\section{CONCLUSIONS}

We have performed exhaustive MD calculations at the $a b$ initio level for $\mathrm{a} \mathrm{Au}_{38}$ cluster capped by thiols with coverages ranging from 1 up to 32 molecules. We have considered three different core models for the cluster and for each case, the thiols were initially placed in different arrangements-bridge adsorption or in the form of staples. We have investigated the energetics, geometry, electronic, and magnetic structure of the relaxed NPs.

The structural analysis reveals a strong interplay between the core configuration and the coverage; when the number of thiols is small the usual fcc spherical core covered by a mixture of staples and bridge adsorbed thiols gives the most stable structures; at intermediate coverages the most stable NPs correspond to a new tubular core geometry protected mainly by staples; around the optimum 24 thiols coverage we retrieve the bi-icosahedral model of Pei et al. ${ }^{12}$ and, finally, at larger coverages the NPs start to polymerize leading to highly distorted clusters and a substantial decrease in their stability. Surprisingly, the adsorption energy does not saturate even at the largest coverages, which seems at contrast with the experimental findings where such a disbandment of the gold cluster is not found. This may fact may be rationalized by noting that at moderately large coverages the surface of the NP is almost completely covered by the thiols. The close-packing of the molecules may impede the penetration of further thiols in order to extract a gold atom from the core and create a new RS-Au-RS motif. In our MD runs, however, all thiols were initially placed close to a gold atom (i.e., the energy barrier required to cross the coating layer was ignored).

On the electronic part, the charge transfer analysis indicates that, up to about $N_{t h}=20$, charge compensation takes place mainly between the surface and the core gold atoms with the thiols remaining fairly neutral. Beyond this coverage there is a charge transfer from the surface atoms to the thiols so that the final electronic arrangement of the NPs consists of a neutral core surrounded by positively charged gold atoms which are bonded to the negatively charged thiols. The 
Density of States projected (PDOS) on the atoms and/or atomic orbitals (AOs) gives no evidence for the formation of closed shell electronic structures, suggesting that the superatom concept is not applicable to the thiol covered $\mathrm{Au}_{38} \mathrm{NPs}$.

Finally, a trivial very weak ferromagnetism develops in all NPs with and odd number of thiols which is characterized by a very small spin-spin coupling $(J<50 \mathrm{meV})$ completely associated to the SOMO. Only for the clean cluster or at very large coverages we seldom find spin splittings in NPs with an even number of thiols due to the existence degenerate MOs at the Fermi level. These results demonstrate that the NPs have no intrinsic ferromagnetic character and therefore, more complex scenarios ${ }^{33}$ need to be further investigated in order to unravel the intriguing magnetic properties of the capped gold NPs.

\section{ACKNOWLEDGMENTS}

This research was supported by the Spanish Ministry of Innovation and Science under Project No. MAT2010-18432.

${ }^{1}$ M.-Ch. Daniel and D. Astruc, Chem. Rev. 104, 293 (2004).

${ }^{2}$ Q. A. Pankhurst, J. Connolly, S. K. Jones, and J. Dobson, J. Phys. D: Appl. Phys. 36, R167 (2003).

${ }^{3}$ O. V. Salata, J. NanoBiotechnology 2, 3 (2004).

${ }^{4}$ S. D. Bader, Rev. Mod. Phys. 78, 1 (2006).

${ }^{5}$ P. D. Jadzinsky, G. Calero, C. J. Ackerson, D. A. Bushnell, and R. D. Kornberg, Science 318, 430 (2007).

${ }^{6}$ M. Zhu, C. M. Aikens, F. J. Hollander, J. Hollander, G. C. Schatz, and R. Jin, J. Am. Chem. Soc. 130, 5883 (2008).

${ }^{7}$ P. Maksymovych, D. C. Sorescu, and J. T. Yates, Jr., Phys. Rev. Lett. 97, 146103 (2006)

${ }^{8}$ H. Häkkinen, R. N. Barnett, and U. Landman, Phys. Rev. Lett. 82, 3264 (1999).

${ }^{9}$ D. E. Jiang, M. L. Tiago, W. Luo, and S. Dai, J. Am. Chem. Soc. 130, 2777 (2008).

${ }^{10}$ D. E. Jiang, W. Luo, M. L. Tiago, and S. Dai, J. Phys. Chem. 112, 13905 (2008).

${ }^{11}$ H. Häkkinen, M. Walter, and H. Gronbeck, J. Phys. Chem. B 110, 9927 (2006).

${ }^{12}$ Y. Pei, Y. Gao, and X. Ch. Zeng, J. Am. Chem. Soc. 130, 7830 (2008).

${ }^{13}$ X. Xing, B. Yoon, U. Landman, and J. H. Parks, Phys. Rev. B 74, 165423 (2006).

${ }^{14}$ I. L. Garzón, K. Michaelian, M. R. Beltrán, A. Posada-Amarillas, P. Ordejón, E. Artacho, D. Sánchez-Portal, and J. M. Soler, Phys. Rev. Lett. 81, 1600 (1998)

${ }^{15}$ C. L. Cleveland, U. Landman, T. G. Schaaff, M. N. Shafigullin, P. W. Stephens, and R. L. Whetten, Phys. Rev. Lett. 79, 1873 (1997).

${ }^{16}$ D. E. Jiang and M. Walter, Phys. Rev. B 84, 193402 (2011).

${ }^{17}$ Y. Pei and X. Ch. Zeng, Nanoscale 4, 4054 (2012).
${ }^{18}$ M. Walter, J. Akola, O. Lope-Acevedo, P. D. Jadzinsky, G. Guillermo Calero, C. J. Ackerson, R. L. Whetten, H. Gronbeck, and H. Häkkinen, Proc. Natl. Acad. Sci. U.S.A. 105, 9157 (2008).

${ }^{19}$ M. Walter, M. Moseler, R. L. Whetten, and H. Häkkinen, Chem. Sci. 2, 1583 (2011)

${ }^{20}$ P. Zhang and T. K. Sham, Appl. Phys. Lett. 81, 736 (2002).

${ }^{21}$ G. L. Nealon, B. Donnio, R. Greget, J.-P. Kappler, E. Terazzi, and J.-L. Gallani, Nanoscale 4, 5244 (2012).

${ }^{22}$ P. Crespo, R. Litrán, T. C. Rojas, M. Multigner, J. M. de la Fuente, J. C. Sánchez-López, M. A. García, A. Hernando, S. Penadés, and A. Fernández, Phys. Rev. Lett. 93, 087204 (2004).

${ }^{23}$ Y. Yamamoto, T. Miura, M. Suzuki, N. Kawamura, H. Miyagawa, T. Nakamura, K. Kobayashi, T. Teranishi, and H. Hori, Phys. Rev. Lett. 93, 116801 (2004).

${ }^{24}$ P. de la Presa, M. Multigner, J. de la Venta, M. A. Garcia, and M. L. RuizGonzalez, J. Appl. Phys. 100, 123915 (2006).

${ }^{25}$ B. Donnio, P. García-Vázquez, J.-L. Gallani, D. Guillon, and E. Terazzi, Adv. Mater. 19, 3534 (2007)

${ }^{26}$ J. De La Venta, E. F. Pinel, M. A. Garcia, P. Crespo, and A. Hernando, Mod. Phys. Lett. B 21, 303 (2007)

${ }^{27}$ J. S. Garitaonandia, M. Insausti, E. Goikolea, M. Suzuki, J. D. Cashion, N. Kawamura, H. Ohsawa, I. Gil de Muro, K. Suzuki, F. Plazaola, and T. Rojo, Nano Lett. 8, 661 (2008).

${ }^{28}$ H. Hori, Y. Yamamoto, T. Iwamoto, T. Miura, T. Teranishi, and M. Miyake, Phys. Rev. B 69, 174411 (2004).

${ }^{29}$ C. González, Y. Simón-Manso, M. Marquez, and V. Mujica, J. Phys. Chem. B 110, 687 (2006).

${ }^{30}$ A. Hernando, P. Crespo, M. A. García, E. Fernández Pinel, J. de la Venta, A. Fernández, and S. Penadés, Phys. Rev. B 74, 052403 (2006).

${ }^{31}$ A. Hernando, P. Crespo, M. A. García, M. Coey, A. Ayuela, and P. M. Echenique, Phys. Status Solidi 248, 2352 (2011).

${ }^{32}$ A. Ayuela, P. Crespo, M. A. García, A. Hernando, and P. M. Echenique, New J. Phys. 14, 013064 (2012).

${ }^{33}$ R. Gréget, G. L. Nealon, B. Vileno, P. Turek, C. Mény, F. Ott, A. Derory, E. Voirin, E. Rivière, A. Rogalev, F. Wilhelm, L. Joly, W. Knafo, G. Ballon, T. Emmanuel, J.-P. Kappler, B. Donnio, and J.-L. Gallani, Chem. Phys. Chem. 13, 3092 (2012).

${ }^{34}$ R. Cuadrado and J. I. Cerdá, J. Phys.: Condens. Matter 24, 086005 (2012).

${ }^{35}$ J. M. Soler, E. Artacho, J. D. Gale, A. García, J. Junquera, P. Ordejón, and D. Sánchez-Portal, J. Phys.: Condens. Matter 14, 2745 (2002).

${ }^{36}$ D. M. Ceperley and B. J. Alder, Phys. Rev. Lett. 45, 566 (1980).

${ }^{37}$ N. Troullier and J. L. Martins, Phys. Rev. B 43, 1993 (1991).

${ }^{38}$ J. P. Perdew, K. Burke, and M. Ernzerhof, Phys. Rev. Lett. 77, 3865 (1996).

${ }^{39}$ F. Ortmann and F. Bechstedt, Phys. Rev B 73, 205101 (2006).

${ }^{40}$ R. Cuadrado, J. I. Cerdá, Y. Wang, G. Xin, R. Berndt, and H. Tang, J. Chem. Phys. 133, 154701 (2010).

${ }^{41}$ C. Hobbs, L. Kantorovich, and J. D. Gale, Surf. Sci. 591, 45 (2005).

${ }^{42}$ P. Mori-Sánchez, A. J. Cohen, and W. Yang, Phys. Rev. Lett. 100, 146401 (2008).

${ }^{43}$ J. I. Cerdá, M. A. Van Hove, P. Sautet, and M. Salmerón, Phys. Rev. B 56, 15885 (1997).

${ }^{44}$ J. I. Cerdá, see http://www.icmm.csic.es/jcerda/ for a description of the GREEN code.

${ }^{45}$ S. G. Louie, S. Froyen, and M. L. Cohen, Phys. Rev. B 26, 1738 (1982). 